\title{
Capacitação da equipe de saúde da família para o cuidado com a saúde auditiva da criança
}

Family health team's training for child's hearing health care Capacitación del equipo de salud familiar para el cuidado de la salud auditiva infantil

Mariane Amaral ${ }^{1}$ Cristiana Magni ${ }^{2}$

${ }^{1}$ Fonoaudióloga, Mestre e doutoranda em Desenvolvimento Comunitário pela Universidade Estadual do Centro-Oeste (UNICENTRO). Professora colaboradora do Departamento de Fonoaudiologia da UNICENTRO, na área de audiologia. E-mail: marianeamaral01@gmail.com, Orcid: http://orcid.org/0000-0001-8501-8186

2 Fonoaudióloga, Doutora pela Universidade Federal do Paraná (UFPR), Docente do Departamento de Fonoaudiologia e do Programa de PósGraduação em Desenvolvimento Comunitário da Universidade Estadual do Centro-Oeste (UNICENTRO). E-mail: crismagni@unicentro.br, Orcid: http://orcid.org/0000-0001-5412-8411 
Resumo: A deficiência auditiva pode manifestar-se tardiamente em crianças de risco para essa privação sensorial de forma que limitar-se somente à Triagem Auditiva Neonatal pode restringir as possibilidades de identificação precoce da deficiência, bem como de realização da intervenção o quanto antes. A habilitação da Equipe de Saúde da Família para o monitoramento das crianças de risco é uma estratégia viável e prevista nas Diretrizes de Atenção da Triagem Auditiva Neonatal. Esta pesquisa teve como objetivo verificar quais metodologias têm sido utilizadas para capacitar a Equipe de Saúde da Família para o cuidado com a audição da criança. Os resultados encontrados apresentam foco nos Agentes Comunitários de Saúde, salientando o seu potencial na identificação de alterações auditivas na população infantil. As capacitações têm sido realizadas de forma presencial e a distância. As ferramentas de ensino a distância são eficazes, contudo o aprendizado de forma presencial continua sendo a metodologia mais efetiva.

Palavras-chave: capacitação; audição; saúde da família; revisão.

\begin{abstract}
Hearing impairment cannot be not detected until well beyond the newborn period in children with risk indicator and the Screening hearing in newborns creates an opportunity, but it does not guarantee optimal outcomes of early identification of the disability as well as the intervention being performed as soon as possible. Enabling the Family Health Team to monitor children at risk is a viable and predictable strategy in the Neonatal Hearing Screening Guidelines. This research aimed to verify which methodologies have been used to enable the Family Health Team to care for the child's hearing. The results show a focus on the Community Health Agents, highlighting their potential in the identification of auditory alterations in the child population. The trainings have been carried out in person and at a distance. Distance learning tools are effective but learning in person continues to be the most effective methodology.
\end{abstract}

Keywords: training; hearing; family health; review.

Resumen: La deficiencia auditiva se puede manifestar tardíamente en infantes de riesgo, de forma que la limitación a, únicamente, el Triaje Auditivo Neonatal puede restringir las posibilidades de identificación precoz de la deficiencia y de una intervención inmediata. La habilitación del Equipo de Salud Familiar para el monitoreo de los infantes en riesgo es una estrategia viable e indicada en las Directrices de Atención al Triaje Auditivo Neonatal. Esta investigación busca verificar las metodologías que han sido utilizadas para capacitar al Equipo de Salud Familiar para el cuidado de la audición infantil. Los resultados encontrados resaltan a los Agentes Comunitarios de Salud, promoviendo su potencial en la identificación de alteraciones auditivas. Las capacitaciones fueron realizadas presencialmente y a distancia. Las herramientas de educación a distancia son eficaces, sin embargo, la educación de forma presencial continúa siendo la metodología más efectiva.

Palabras clave: capacitación; audición; salud familiar; revisión. 


\section{INTRODUÇÃO}

A integridade anatômica e funcional do órgão da audição está diretamente relacionada ao desenvolvimento da fala, de forma que a privação auditiva nos primeiros anos de vida irá impactar significativamente o desenvolvimento psicossocial da criança.

A Lei Federal n. 12.303 (BRASIL, 2010) tornou obrigatória a realização do teste da orelhinha como estratégia para a identificação de alterações auditivas em recém-nascidos. Todavia a deficiência auditiva pode manifestar-se tardiamente em crianças de risco para tal privação sensorial e, por conseguinte, limitar-se somente à triagem auditiva neonatal restringe as possibilidades de identificação precoce da deficiência nessa população. Uma estratégia para a ampliação da detecção em diferentes níveis de atenção à saúde é o monitoramento das crianças do grupo de risco. De acordo com as Diretrizes de Atenção da Triagem Auditiva Neonatal, os neonatos e lactentes com risco para deficiência auditiva, mesmo aqueles que obtiveram respostas satisfatórias na triagem, devem realizar o monitoramento mensal do desenvolvimento da audição e da linguagem na atenção básica.

Conforme a Política Nacional de Atenção Básica, as ações desenvolvidas no âmbito da equipe Estratégia da Saúde da Família (ESF) devem ser prioritárias para expansão e consolidação da atenção básica. Entre os profissionais da ESF destaca-se o Agente Comunitário de Saúde (ACS), o qual atua como elo entre as necessidades de saúde da comunidade e a melhoria das condições de vida local.

Diante disso, a habilitação dos profissionais da ESF para realizar o monitoramento das crianças de risco para a deficiência auditiva é viável e deve possibilitar a identificação precoce da perda auditiva, bem como o encaminhamento adequado para o diagnóstico e intervenção. Logo, para que esses profissionais de saúde estejam capacitados na área do cuidado com a audição da população infantil, é preciso que haja educação em saúde auditiva. Essa educação ou capacitação requer fontes consistentes de informação para que os profissionais possam construir seu conhecimento, imprimir significado a este e incorporá-lo com segurança em sua prática profissional. 
Entretanto as atividades de capacitação não podem ser pontuais e isoladas, havendo a necessidade da educação permanente para esses profissionais, não só no que se refere à saúde auditiva, mas nas diferentes áreas da saúde.

Nesse contexto, o objetivo deste estudo é verificar quais metodologias têm sido utilizadas para capacitar os profissionais da ESF para o cuidado com a audição da criança.

\section{MATERIAIS E MÉTODOS}

Trata-se de uma revisão integrativa da literatura, a qual objetivou, por meio de uma metodologia sistemática de busca, seleção e análise, descrever a produção científica desta temática, destacando o estado da arte e apresentando as possibilidades de futuras investigações (ESPOTE; SERRALHA; SCORSOLINI-COMIN, 2013).

A elaboração dessa revisão constou de seis etapas, sendo elas: (1) identificação do tema e seleção da questão de pesquisa; (2) estabelecimento de critérios para inclusão e exclusão de estudos; (3) definição das informações a serem extraídas dos estudos selecionados; (4) avaliação dos estudos incluídos na revisão integrativa; (5) interpretação dos resultados e (6) apresentação da síntese do conhecimento (MENDES; SILVEIRA; GALVÃO, 2008).

A questão norteadora estabelecida foi: quais metodologias têm sido utilizadas para capacitar os profissionais da ESF com relação ao cuidado com a audição da criança?

A busca foi realizada nas bases de dados eletrônicas SciELO (através do site http://www.scielo.org), Medline e Lilacs (através do Portal Regional da Biblioteca Virtual em Saúde - BVS, disponível no endereço: http://bvsalud. org/), e busca na literatura não indexada (Google Acadêmico, disponível no endereço: https://scholar.google.com.br/). Os descritores utilizados foram os termos "capacitação", "audição" e "saúde da família" e seus correspondentes em inglês "training", "hearing" e "family health". Todos esses descritores pertencem ao Descritores em Ciências da Saúde (DeCS) e foram utilizados em combinação usando o operador booleano AND.

Foram elencados como critérios de inclusão: textos disponíveis gratuitamente na íntegra em meio eletrônico, nos idiomas português ou inglês, 
publicados em periódicos nacionais e internacionais. Não foi delimitado ano de publicação para os estudos.

Foram utilizados como critério de exclusão: artigos de revisão de literatura, trabalhos de conclusão de curso, dissertações, teses e estudos cujas metodologias de pesquisa não se constituíram de capacitações para profissionais da ESF no âmbito da saúde auditiva infantil.

Para a avaliação dos estudos científicos levantados, na busca online, foram estabelecidos critérios contemplando os aspectos: profissionais da amostra do estudo, forma de avaliação da capacitação, origem do material didático utilizado, metodologia da capacitação e resultados obtidos.

\section{RESULTADOS E DISCUSSÃO}

Na base de dados SciELO, foram encontrados 22 resultados e, no portal da BVS, 39 resultados. No Google Acadêmico, foram analisadas as 50 primeiras referências organizadas de acordo com o nível de relevância. A figura 1 apresenta a síntese do processo de localização e seleção dos estudos. Após a leitura dos estudos selecionados, apenas quatro foram incluídos na etapa final desta revisão de literatura (Quadro 1). A síntese dos critérios avaliados nos estudos científicos selecionados é apresentada no Quadro 2.

Figura 1-Síntese do processo de localização dos estudos selecionados para a revisão integrativa da literatura

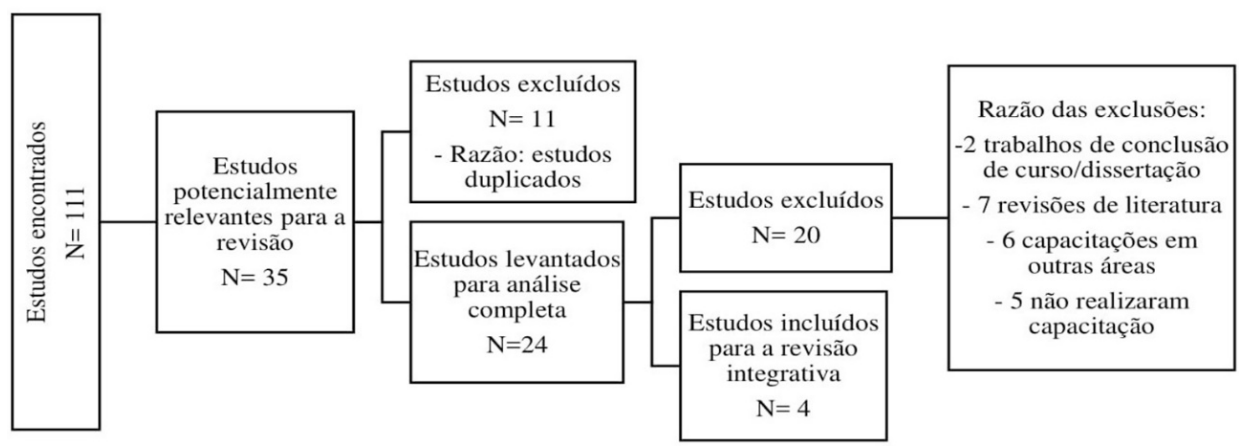

Fonte: Elaborada pelas autoras. 
Quadro 1 - Síntese dos estudos incluídos na revisão integrativa de literatura

\begin{tabular}{|c|c|c|c|c|c|}
\hline Estudo & Ano & Autor & \begin{tabular}{|l|} 
Periódico \\
Científico \\
\end{tabular} & Título & Objetivo \\
\hline Artigo 1 & 2008 & $\begin{array}{l}\text { Alvarenga } \\
\text { et al. }\end{array}$ & $\begin{array}{c}\text { Pró-Fono } \\
\text { R. Atual. } \\
\text { Cient. }\end{array}$ & $\begin{array}{l}\text { Proposta para } \\
\text { capacitação } \\
\text { de agentes } \\
\text { comunitários de } \\
\text { saúde em saúde } \\
\text { auditiva }\end{array}$ & $\begin{array}{l}\text { Organizar e verificar } \\
\text { a efetividade de } \\
\text { um programa de } \\
\text { capacitação de agentes } \\
\text { comunitários de saúde } \\
\text { do Programa de Saúde } \\
\text { da Família, na área de } \\
\text { saúde auditiva infantil }\end{array}$ \\
\hline Artigo 2 & 2010 & $\begin{array}{l}\text { Melo } \\
\text { et al. }\end{array}$ & $\begin{array}{l}\text { Pró-Fono } \\
\text { R. Atual. } \\
\text { Cient. }\end{array}$ & $\begin{array}{l}\text { Capacitação } \\
\text { de agentes } \\
\text { comunitários } \\
\text { de saúde em } \\
\text { saúde auditiva: } \\
\text { efetividade da } \\
\text { videoconferência }\end{array}$ & $\begin{array}{l}\text { Avaliar a efetividade } \\
\text { da capacitação dos } \\
\text { agentes comunitários } \\
\text { de saúde, por meio da } \\
\text { videoconferência, na } \\
\text { área de saúde auditiva } \\
\text { infantil }\end{array}$ \\
\hline Artigo 3 & 2013 & $\begin{array}{l}\text { Araújo } \\
\text { et al. }\end{array}$ & $\begin{array}{l}\text { Int. J } \\
\text { Audiol }\end{array}$ & $\begin{array}{l}\text { Community } \\
\text { health worker } \\
\text { training for infant } \\
\text { hearing health: } \\
\text { Effectiveness of } \\
\text { distance learning }\end{array}$ & $\begin{array}{l}\text { Avaliar a efetividade } \\
\text { de um programa de } \\
\text { capacitação a distância } \\
\text { em saúde auditiva } \\
\text { infantil para agentes } \\
\text { comunitários de saúde }\end{array}$ \\
\hline Artigo 4 & 2017 & $\begin{array}{l}\text { Bernardi } \\
\text { et al. }\end{array}$ & Rev. CEFAC & $\begin{array}{l}\text { O uso da CIF no } \\
\text { acompanhamento } \\
\text { do desenvolvimento } \\
\text { auditivo e de } \\
\text { linguagem de } \\
\text { crianças no } \\
\text { primeiro ano de } \\
\text { vida }\end{array}$ & $\begin{array}{l}\text { Utilizar a Classificação } \\
\text { Internacional de } \\
\text { Funcionalidade, } \\
\text { Incapacidade e Saúde } \\
\text { - Crianças e Jovens } \\
\text { (CIF-CJ) no registro do } \\
\text { desenvolvimento da } \\
\text { audição e da linguagem } \\
\text { de crianças no primeiro } \\
\text { ano de vida em um } \\
\text { serviço de atenção } \\
\text { básica }\end{array}$ \\
\hline
\end{tabular}

Fonte: Elaborada pelas autoras.

O Ministério da Saúde instituiu a Política Nacional de Educação Permanente em Saúde (EPS) como forma de promover a formação e o desenvolvimento de habilidades dos profissionais da ESF (BRASIL, 2007). 
Contudo os resultados desta revisão de literatura mostram escassez de atividades de formação em saúde auditiva infantil para a ESF.

Considerando o impacto da deficiência auditiva não só para o desenvolvimento da fala da criança, mas o seu desenvolvimento psicossocial na ausência de uma intervenção, é possível inferir a existência da falta de valorização da área em nível nacional. A habilitação da ESF pode contribuir significativamente na diminuição das dificuldades supracitadas, uma vez que a literatura aponta modificação positiva do desfecho clínico da deficiência auditiva quando a identificação e a intervenção acontecem de forma precoce (COSTA et al., 2017; AMEMIYA; GOULART; CHIARI, 2013).

Quadro 2 - Síntese dos critérios avaliados nos estudos selecionados para a etapa final da revisão de literatura

\begin{tabular}{|c|c|c|c|c|}
\hline & Artigo 1 & Artigo 2 & Artigo 3 & Artigo 4 \\
\hline $\begin{array}{l}\text { Profissionais } \\
\text { da amostra }\end{array}$ & ACS & ACS & ACS & ACS \\
\hline $\begin{array}{l}\text { Forma de } \\
\text { avaliação da } \\
\text { capacitação }\end{array}$ & $\begin{array}{l}\text { Questionário }{ }^{1} \\
\text { pré e pós } \\
\text { capacitação }\end{array}$ & $\begin{array}{l}\text { Questionário }{ }^{1} \\
\text { pré e pós } \\
\text { capacitação }\end{array}$ & \begin{tabular}{|l|} 
Questionário \\
pré e pós \\
capacitação e \\
simulador de \\
situações diárias \\
aplicado seis \\
meses após a \\
ação
\end{tabular} & $\begin{array}{l}\text { Questionário } \\
\text { pré e pós } \\
\text { capacitação }\end{array}$ \\
\hline $\begin{array}{l}\text { Origem do } \\
\text { material } \\
\text { didático } \\
\text { utilizado }\end{array}$ & $\begin{array}{l}\text { Adaptação" } \\
\text { do material } \\
\text { "Primary ear } \\
\text { and hearing } \\
\text { care training } \\
\text { resource" }\end{array}$ & $\begin{array}{l}\text { Adaptação }{ }^{1} \\
\text { do material } \\
\text { "Primary ear } \\
\text { and hearing } \\
\text { care training } \\
\text { resource" }\end{array}$ & $\begin{array}{l}\text { Adaptação }{ }^{1} \\
\text { do material } \\
\text { "Primary ear } \\
\text { and hearing } \\
\text { care training } \\
\text { resource" }\end{array}$ & $\begin{array}{l}\text { Adaptação } \\
\text { do material } \\
\text { "Primary ear } \\
\text { and hearing } \\
\text { care training } \\
\text { resource" }\end{array}$ \\
\hline $\begin{array}{l}\text { Metodologia } \\
\text { da } \\
\text { capacitação }\end{array}$ & $\begin{array}{l}\text { Aula } \\
\text { expositiva } \\
\text { presencial }\end{array}$ & $\begin{array}{l}\text { Aula expositiva } \\
\text { presencial e } \\
\text { videoconferência } \\
\text { simultânea }\end{array}$ & CD-ROM & $\begin{array}{l}\text { Oficinas } \\
\text { presenciais }\end{array}$ \\
\hline
\end{tabular}




\begin{tabular}{|c|c|c|c|c|}
\hline & Artigo 1 & Artigo 2 & Artigo 3 & Artigo 4 \\
\hline $\begin{array}{l}\text { Resultados } \\
\text { obtidos }\end{array}$ & $\begin{array}{l}\text { Aumento no } \\
\text { escore total } \\
\text { obtido no } \\
\text { questionário } \\
\text { pós- } \\
\text {-capacitação }\end{array}$ & $\begin{array}{l}\text { Nível } \\
\text { semelhante } \\
\text { de assimilação } \\
\text { do conteúdo } \\
\text { ministrado em } \\
\text { grupos que } \\
\text { participaram } \\
\text { da mesma } \\
\text { capacitação, } \\
\text { mas por } \\
\text { metodologias } \\
\text { diferentes }\end{array}$ & $\begin{array}{l}\text { Quantidade } \\
\text { significante de } \\
\text { informações } \\
\text { retida no } \\
\text { momento pós- } \\
\text {-capacitação } \\
\text { imediato e } \\
\text { incorporação } \\
\text { dos conteúdos } \\
\text { teóricos } \\
\text { nas ações } \\
\text { que os Acs } \\
\text { desenvolvem } \\
\text { diariamente }\end{array}$ & $\begin{array}{l}\text { Aumento no } \\
\text { escore total } \\
\text { obtido no } \\
\text { questionário } \\
\text { pós- } \\
\text {-capacitação }\end{array}$ \\
\hline
\end{tabular}

ACS: Agente Comunitário de Saúde.

${ }^{1}$ Materiais adaptados por Alvarenga et al. (2008) a partir de publicação da Organização Mundial da Saúde (WHO, 2006).

Fonte: Elaborada pelas autoras.

Nos artigos 1 e 2, os ACS não possuíam experiência prévia em saúde auditiva infantil no momento da capacitação; já no artigo 3, participaram dois grupos de ACS, sendo que um grupo já havia participado anteriormente de atividades de capacitação envolvendo saúde auditiva e o outro grupo não havia recebido capacitação prévia na área e, no artigo 4, não havia informação clara a respeito da existência de formação prévia dos ACS na área de saúde auditiva. O ACS é um profissional que atua na educação em saúde, participando da organização do trabalho coletivo, incluindo o planejamento e a execução das ações interdisciplinares e a educação popular em saúde (BRASIL, 2016). Wagner et al. (2017) afirmam que esses profissionais são ideais para a implantação de programas de monitoramento da saúde auditiva maternoinfantil na atenção básica. O direcionamento das capacitações para os ACS evidencia a valorização das potencialidades de atuação desses profissionais. Com base na articulação de diferentes políticas e normativas que sustentam a operacionalização do SUS, é sustentada a consideração do importante papel dos ACS nas equipes de saúde e o reconhecimento de necessidades específicas de educação permanente desses agentes (BRASIL, 2016). 
Nos artigos 1 e 2, além de verificar o desempenho dos ACS nos questionários, os autores também apresentaram comentários e depoimentos dos participantes sobre a importância da capacitação e o impacto desta em sua atuação profissional (ALVARENGA et al., 2008; MELO et al., 2010). O amplo uso de questionários para avaliação de ações de ensino em saúde possibilita mensurar impactos da metodologia de ensino-aprendizagem utilizada. Gerhardt e Silveira (2009) citam, entre as vantagens do uso de questionários, a obtenção de respostas mais rápidas e mais precisas e ainda permite mais uniformidade na avaliação, em virtude da natureza impessoal do instrumento. Entre as desvantagens, uma questão pode influenciar outra quando é feita a leitura de todas as perguntas antes do início das respostas. Dessa forma, o uso de questionários para avaliação de ações de capacitação e mensuração da informação assimilada é plausível, permitindo análise adequada do processo realizado. Tanto os questionários quanto os materiais didáticos utilizados na capacitação dos ACS envolvem os temas: (1) audição e deficiência auditiva, (2) tipos, prevenção e causas da deficiência auditiva, (3) técnicas de identificação e diagnóstico da deficiência auditiva e (4) aspectos gerais da deficiência auditiva.

No que se refere aos materiais didáticos utilizados, a adaptação de uma apostila permitiu que os ACS acompanhassem as atividades realizadas de forma interativa (ALVARENGA et al., 2008; MELO et al., 2010). O CD-ROM utilizado por Araújo et al. (2013) foi intitulado "Saúde Auditiva Infantil" e foi desenvolvido previamente em parceria do Departamento de Fonoaudiologia da Faculdade de Odontologia de Bauru com a Disciplina de Telemedicina da Faculdade de Medicina da Universidade de São Paulo. A capacitação oferecida aos ACS no estudo de Bernardi et al. (2017) envolveu um maior número de temas, uma vez que também treinou esses profissionais para aplicação do 'Questionário para monitoramento do desenvolvimento auditivo e de linguagem no primeiro ano de vida', desenvolvido por Alvarenga et al. (2013).

O material didático utilizado para capacitar profissionais de saúde deve apresentar embasamento teórico adequado e oferecer informações especificas da área da audição. O conhecimento de questões especificas dos estudos da audição pode auxiliar na identificação de alterações auditivas, principalmente daquelas comuns em crianças com outras patologias 
associadas. Barbosa et al. (2013) afirmam que, apesar do conhecimento sobre tipos e etiologias da deficiência auditiva ser específico dos profissionais que lidam com a audição, esse aspecto possibilita ao profissional de saúde um olhar diferenciado sobre as crianças que apresentem determinadas etiologias associadas a perdas auditivas com tipos e graus específicos.

No que concerne às metodologias utilizadas para a formação dos ACS, as capacitações foram presenciais e à distância. No estudo que utilizou o CD-ROM, a capacitação foi individual, ou seja, cada participante avançou pela capacitação em seu ritmo, e, portanto, todos os participantes receberam um treinamento em informática básica antes do início da capacitação propriamente dita, para que todos tivessem as habilidades mínimas necessárias para o uso do computador e da ferramenta disponibilizada.

Analisando esses estudos, diferentes ferramentas de ensino foram utilizadas para capacitar os ACS. A utilização de ferramentas de ensino a distância possibilitou o alcance a grupos de ACS geograficamente distantes, o que Ihes possibilitou receber formação adequada sobre o cuidado com a audição da criança. A situação do setor de saúde brasileiro é complexa, e a utilização de ferramentas para a diminuição das desigualdades no território nacional representa a oportunidade para aumentar a eficiência dos serviços de saúde (CONCEIÇÃO; BARREIRA-NIELSEN, 2014). Dessa forma, as capacitações à distância tornam-se uma ferramenta viável para a capacitação dos profissionais da ESF. O ensino à distância ganhou espaço por suas potencialidades e vêm se ampliando desde 2007, em virtude da implantação do Programa Nacional de Telessaúde. A atenção primária à saúde já tem utilizado recursos tecnológicos de informação e comunicação, no intuito de apoiar os profissionais de saúde, qualificar a assistência e possibilitar mudanças no processo de trabalho das equipes de saúde (CASTRO; ZUCKI, 2015). Nascimento et al. (2017) verificaram que atividades de tele-educação por webconferência constituem um instrumento ativo de propagação do conhecimento que pode fortalecer o processo de educação permanente dos profissionais que atuam na atenção primária à saúde.

Outros estudos relevantes na área de saúde auditiva infantil e/ou capacitação de profissionais de saúde, mas que não se adequavam aos critérios desta revisão integrativa, mostram que, em se tratando de ensino à 
distância, cerca de $67 \%$ dos participantes afirmam se sentir confortáveis em participar de uma webconferência, mas apenas 35\% dos ACS acreditam que a atividade online seja satisfatória quando comparada a uma aula presencial (CONCEIÇÃO; BARREIRA-NIELSEN, 2014).

Quanto aos resultados obtidos, a capacitação foi considerada satisfatória quando o desempenho no escore total do questionário esteve acima de $70 \%$ de acertos após a capacitação, sendo que os quatro estudos consideraram suas intervenções como satisfatórias (ALVARENGA et al., 2008; MELO et al., 2010; ARAÚJO et al., 2013; BERNARDI et al., 2017). Tal dado assemelha-se ao encontrado em outras populações capacitadas na área de saúde auditiva infantil, como o estudo de Barbosa et al. (2013), que observaram mudança significativa de conhecimento dos profissionais de enfermagem após ação educativa na área.

Independente da metodologia de ensino utilizada, a capacitação dos ACS é efetiva para a aquisição do conhecimento em saúde auditiva, contudo o aprendizado de forma presencial continua sendo a metodologia mais efetiva (MELO et al., 2010; 2011). De acordo com Melo et al., 2011, isso ocorre devido ao controle da interação entre os participantes, bem como a motivação, a atenção e o interesse deles, sendo essas condições importantes para o aprendizado, o que difere da videoconferência para a obtenção deste feedback do público pelo ministrante. Além disso, a utilização de uma apostila como material de apoio possibilita uma capacitação dos ACS mais eficaz, permitindo a compreensão de temas que não são inerentes à sua prática (ALVARENGA et al., 2008). Bernardi et al. (2017) afirmam que a capacitação dos ACS em saúde auditiva infantil permitiu a ampliação do olhar sobre aspectos do desenvolvimento infantil favorecendo eventuais intervenções necessárias.

Para Arakawa et al. (2013), a formação oferecida aos ACS fornece subsídios para formar recursos humanos e transmitir conhecimentos aos agentes para que possam atuar junto à comunidade, proporcionando-lhe melhoras na qualidade de vida. Pesquisadores mapearam o conhecimento de ACS, antes e após participação em oficinas de instrumentalização sobre aspectos fonoaudiológicos, e verificaram que um número maior de ACS passou a considerar a relevância da observação da atenção auditiva em 
bebês ou de considerar a queixa dos pais acerca de questões auditivas (LOPES et al., 2015).

Diante de tais evidências, ressalta-se a importância de capacitar os ACS da ESF e de como essas capacitações são eficientes, pois os profissionais passam a direcionar o olhar com maior consideração sobre os aspectos da audição e linguagem oral. A ESF da atenção básica deve ser o contato preferencial dos usuários, a principal porta de entrada e centro de comunicação da rede de atenção à saúde (BRASIL, 2011), por isso a necessidade de que esses profissionais estejam aptos para atuar no cuidado com a audição das crianças.

Nos estudos encontrados, apenas um fez avaliação a médio prazo pós-capacitação, todos os outros fizeram análise da assimilação do conteúdo no momento pós-capacitação imediato. Isso leva à reflexão sobre o acompanhamento dos trabalhos desses profissionais na área de audição. Mais estudos podem ser feitos com profissionais que já foram capacitados, a fim de verificar o conhecimento deles e como a formação tem sido aplicada na prática profissional decorridos meses ou mesmo anos da primeira capacitação. Dessa forma, também se torna possível promover atualização de conhecimento para esses profissionais e contribuir para o processo de educação permanente em saúde. Um estudo realizado por Araújo et al. (2015) capacitou ACS por meio de um Cybertutor, sistema de ensino baseado na web, e aplicou questionário de avaliação imediatamente após a capacitação e, novamente, o mesmo questionário decorridos 15 meses da capacitação. Os autores verificaram redução significante, em percentual, do conhecimento geral dos ACS sobre saúde auditiva infantil, demonstrando a importância da educação continuada para esses profissionais.

A educação permanente, além da sua evidente dimensão pedagógica, deve ser encarada também como uma importante estratégia de gestão, com grande potencial provocador de mudanças no cotidiano dos serviços em sua micropolítica, bastante próximo dos efeitos concretos das práticas de saúde na vida dos usuários, e como um processo que se dá "no trabalho, pelo trabalho e para o trabalho" (BRASIL, 2011). A política nacional de EPS (BRASIL, 2007) prevê que as secretarias estaduais e municipais de saúde são responsáveis por planejar a formação e a educação permanente de 
trabalhadores em saúde necessários ao SUS no seu âmbito de gestão e, além disso, articular, junto às instituições de ensino técnico e universitário, mudanças em seus cursos técnicos, de graduação e pós-graduação de acordo com as necessidades do SUS, estimulando uma postura de corresponsabilidade sanitária. Vale ressaltar que a capacitação desses profissionais não deve ocorrer em um momento único, mas deve estar alicerçada em uma proposta de educação permanente em saúde, a fim de propiciar maiores possibilidades de retenção da informação e de transformação da realidade em saúde (ARAÚjO et al., 2015).

Ações de capacitação como as propostas nos estudos incluídos nesta revisão de literatura estão de acordo com as diretrizes da portaria de EPS. Ações de educação em saúde, ainda que não sejam projetos permanentes, são um início para a implantação da EPS e um meio para as secretarias estaduais e municipais poderem propor projetos que garantam a EPS para os profissionais do SUS. É imprescindível que o profissional consiga aplicar o conhecimento em suas atividades diárias de atuação não apenas no momento pós-capacitação imediata, mas sim continuamente (ARAúJO et al., 2015).

Diante disso, considerando o pequeno número de ações específicas em saúde auditiva infantil encontradas na literatura, sugere-se que mais estudos possam ser realizados na área, buscando ampliar o conhecimento desses profissionais fundamentais para o sistema de saúde. Havendo um material cujo conteúdo é apropriado para profissionais como ACS, que se encontra traduzido e adaptado para o Português, as capacitações na área do cuidado com a audição da criança podem ser realizadas de forma padronizada no país.

Os estudos incluídos nesta revisão de literatura capacitaram apenas os ACS, e tal fato pode ser observado pelo prisma da valorização desse profissional e reconhecimento da importância do seu papel na atenção à saúde como elo entre o sistema de saúde e a comunidade. Contudo acredita-se que os demais profissionais da ESF também podem e devem ser capacitados na área, ampliando e aprimorando o cuidado com a população infantil. 


\section{CONCLUSÃO}

Quatro estudos foram incluídos nesta revisão, evidenciando escassez de publicações de atividades de capacitação realizadas na área do cuidado com a audição da criança para os profissionais da ESF em nível nacional.

Todos os estudos foram realizados com ACS, salientando o potencial desse profissional na identificação de alterações auditivas na população infantil.

As capacitações foram realizadas de forma presencial com aulas expositivas e oficinas, e à distância na forma de videoconferência e CD-ROM. Todos os estudos consideraram o desempenho pós-capacitação imediato satisfatório, tendo como critério acertos acima de $70 \%$ no escore total do questionário.

\section{REFERÊNCIAS}

ALVARENGA, Kátia de Freitas et al. Questionário para monitoramento do desenvolvimento auditivo e de linguagem no primeiro ano de vida. CoDAS, São Paulo, v. 25, n. 1, p. 16-21, 2013.

ALVARENGA, Kátia Freitas et al. Proposta para capacitação de agentes comunitários de saúde em saúde auditiva. Pró-Fono Revista de Atualização Científica, Barueri, SP, v. 20, n. 3, p. 171-6, jul./set. 2008.

AMEMIYA, Erica Endo; GOULART, Barbara Niegia Garcia; CHIARI, Brasilia Maria. Use of nouns and verbs in the oral narrative of individuals with hearing impairment and normal hearing between 5 and 11 years of age. São Paulo Medical Journal, São Paulo, v. 131, n. 5, p. 289-95, 2013.

ARAKAWA, Aline M. et al. Avaliação de um programa de capacitação em fonoaudiologia para agentes comunitários de saúde na Amazônia brasileira. Distúrbios da Comunicação, São Paulo, v. 25, n. 2, ago. 2013.

ARAÚJO, Eliene Silva et al. Community health worker training for infant hearing health: effectiveness of distance learning. International Journal of Audiology, v. 52, n. 9, p. 636-41, set. 2013.

ARAÚJO, Eliene S.; JACOB-CORTELETTI, Lilian Cassia B.; ABRAMIDES, Dagma V. M.; ALVARENGA, Kátia F. Capacitação de agentes comunitários de saúde na área de 
saúde auditiva infantil: retenção da informação recebida. Revista CEFAC, São Paulo, v. 17, n. 2, p. 445-53, mar./abr. 2015.

BARBOSA, Camila Padilha et al. Educação em saúde auditiva do neonato e lactente para profissionais de enfermagem. Brazilian Journal of Otorhinolaryngology, São Paulo, v. 79, n. 2, p. 226-32, abr. 2013.

BERNARDI, Sarah Alvarenga et al. O uso da CIF no acompanhamento do desenvolvimento auditivo e de linguagem de crianças no primeiro ano de vida. Revista CEFAC, São Paulo, v. 19, n. 2, p. 159-70, mar./abr. 2017.

BRASIL. Ministério da Saúde. Secretaria de Gestão do Trabalho e da Educação na Saúde. Departamento de Gestão da Educação na Saúde. Diretrizes para capacitação de agentes comunitários de saúde em linhas de cuidado. Brasília: Ministério da Saúde, 2016. 46 p.

BRASIL. Ministério da Saúde. Secretaria de Atenção à Saúde. Departamento de Ações Programáticas Estratégicas. Diretrizes de Atenção da Triagem Auditiva Neonatal. Brasília: Ministério da Saúde, 2012. 32 p.

BRASIL. Ministério da Saúde. Portaria n. 2.488, de 21 de outubro de 2011. Aprova a Política Nacional de Atenção Básica, estabelecendo a revisão de diretrizes e normas para a organização da Atenção Básica, para a Estratégia Saúde da Família (ESF) e o Programa de Agentes Comunitários de Saúde (PACS). Diário Oficial da União, Brasília, 2011.

BRASIL. Lei n. 12.303, de 2 de agosto de 2010. Dispõe sobre a obrigatoriedade de realização do exame denominado Emissões Otoacústicas Evocadas. Diário Oficial da União, Brasília, DF, 3 ago. 2010. Seção 1, p 1.

BRASIL. Ministério da Saúde. Portaria n. 1.996, de 20 de agosto de 2007. Dispõe sobre as diretrizes para a implementação da política nacional de educação permanente em saúde e dá outras providências. Diário Oficial da União, Brasília, 2007.

CASTRO, Taís Teixeira de Oliveira; ZUCKI, Fernanda. Capacitação do Agente Comunitário de Saúde na saúde auditiva infantil: perspectivas atuais. CoDAS, São Paulo, v. 27, n. 6, p. 616-22, dez. 2015.

CONCEIÇÃO, Heyder Vieira da; BARREIRA-NIELSEN, Carmen. Capacitação em saúde auditiva: avaliação da ferramenta no Programa de Telessaúde Brasil. Revista CEFAC, São Paulo, v. 16, n. 5, p. 1426-33, set./out. 2014. 
COSTA, Letícia Splendor da; SILVA, Paula Botelho da; AZEVEDO, Marisa Frasson de; GIL, Daniela. Porcentagem de consoantes corretas (PCC) em crianças com deficiência auditiva: estudo longitudinal. Revista CEFAC, São Paulo, v. 19, n. 2, p. 171-9, mar./abr. 2017.

ESPOTE, Roberta; SERRALHA, Conceição Aparecida; SCORSOLINI-COMIN, Fabio. Inclusão de surdos: revisão integrativa da literatura científica. Psico-USF, Itatiba, SP, v. 18, n. 1, p. 77-88, jan./abr. 2013.

GERHARDT, Tatiana Engel; SILVEIRA, Denise Tolfo. Métodos de pesquisa. Porto Alegre, RS: Editora da UFRGS, 2009. 120 p.

LOPES, Nathália de C.; VIEIRA, Gleice Ane S. S.; PENA, Suelle Regina B.; LEMOS, Stela Maris A. Agentes comunitários de saúde: mapeamento de conhecimento antes e após oficinas de instrumentalização. Revista CEFAC, São Paulo, v. 17, n. 3, p. 683-94, maio/jun. 2015.

MELO, Tatiana Mendes de; ALVARENGA, Kátia de Freitas; BLASCA, Wanderléia Quinhoneiro; TAGA, Marcel Frederico de Lima. Capacitação de agentes comunitários de saúde em saúde auditiva: efetividade da videoconferência. Pró-Fono Revista de Atualização Científica, Barueri, SP, v. 22, n. 2, p. 139-44, abr./jun. 2010.

MELO, Tatiana Mendes de; ALVARENGA, Kátia de Freitas; BLASCA, Wanderléia Quinhoneiro; TAGA, Marcel Frederico de Lima. Opinião dos agentes comunitários de saúde sobre o uso da videoconferência na capacitação em saúde auditiva infantil. Revista CEFAC, São Paulo, v. 13, n. 4, p. 692-7, jul./ago. 2011.

MENDES, Karina Dal Sasso; SILVEIRA, Renata Cristina de Campos Pereira; GALVAO, Cristina Maria. Revisão integrativa: método de pesquisa para a incorporação de evidências na saúde e na enfermagem. Texto \& Contexto - Enfermagem, Florianópolis, v. 17, n. 4, p. 758-64, out./dez. 2008.

NASCIMENTO, Cynthia Maria Barboza do et al. Telefonoaudiologia como estratégia de educação permanente na atenção primária à saúde no Estado de Pernambuco. Revista CEFAC, São Paulo, v. 19, n. 3, p. 371-80, maio/jun. 2017.

WAGNER, Julianne; BONAMIGO, Andrea Wander; OLIVEIRA, Fabiana de; MACHADO, Márcia Salgado. Monitoramento da audição e da linguagem na atenção primária à saúde: projeto piloto. Ciência \& Saúde Coletiva, Rio de Janeiro, v. 22, n. 11, p. 3599-606, nov. 2017. 
WORLD HEALTH ORGANIZATION (WHO). Primary ear and hearing care training resource: basic, intermediate and advanced levels. Geneva: World Health Organization, 2006. Disponível em: http://www.who.int/pbd/deafness/activities/ hearing_care/en/ 
